\title{
Crypto-miners: Digital labor and the power of blockchain technology
}

\author{
Filipe Calvão
}

\author{
Graduate Institute of International and Development Studies, 1211, Genève, 1, Switzerland \\ Corresponding author: Filipe Calvão; e-mail: filipe.calvao@graduateinstitute.ch
}

This article examines the labor power of digital miners. Though an obscure and still incipient facet of the digital economy, crypto-mining powers and secures transactions across blockchains, or public distributed digital ledgers. Drawing from interviews with cryptocurrency enthusiasts, blockchain advocates, and developers; participation in online and offline discussions; and a survey with small-scale crypto-miners, this article takes on the material and technoscientific valuation of crypto-mining to understand how a future of open, decentralized accountability implicates human labor alongside automated processes. The work of digital mining, performed in the work of inscribing, registering, and politically organizing mining operations, enables the formation of democratic communities in the digital economy and remains inevitably embedded in social relations as a mode of productive, meaningful action.

Keywords Digital Labor; Blockchain; Cryptocurrency; Mining; Bitcoin; Crypto-mining

\section{The new extractive frontier of digital mining}

\begin{abstract}
The labour of his body, and the work of his hands, we may say, are properly his. Whatsoever then he removes out of the state that nature hath provided, and left it in, he hath mixed his labour with, and joined to it something that is his own, and thereby makes it his property.

- John Locke, Two Treatises of Government
\end{abstract}

In the diamond and gold rush of the late nineteenth century in South Africa, the potential for wealth entailed a claim of ownership over land. Governing the stakes of artisanal mining also propelled radical forms of exercising law and justice, popularly known in South African historiography as a space of "digger's democracy." As soon as the first wave of diamond and gold rushers settled in, capital concentration and the growing technical and engineering complexity of resource extraction gave way to systematic forms of recruitment and labor-intensive mining complexes. If cheap labor and accessible minerals assured the early profitability of the mining industry, seasonal labor shortages and regular strikes were decisive in enforcing a regime of discipline, control, and capital concentration across diamond and gold mining fields. Although only one decade since the inception of the Bitcoin network, ${ }^{1}$ and its underlying blockchain technology, this article suggests that an analogous process is currently taking place with the emergent economy of cryptocurrencies. In fact, the cost of digital mining by the expenditure of computational power and energy can be too high to make mining profitable for small-scale miners; conversely, the high valuation of cryptocurrencies has precipitated a flood of capital-intensive, industrial miners with higher computing power, pushing small-scale miners out of the system of incentives in place.

As a trained gemologist and anthropologist with ethnographic research in diamond mines in Africa, I have long been intrigued by digital mining, the bedrock of the cryptocurrency ecosystem. The original comparison with gold mining is laid out in the founding document of the Bitcoin technology to account for the process of mining bitcoins: "The steady addition of a constant of $[$ sic $]$ amount of new coins," the mythical figure of Satoshi Nakamoto $(2008,4)$ 
wrote, "is analogous to gold miners expending resources to add gold to circulation. In our case, it is CPU time and electricity that is expended." Despite attempts to trace the historical depth of this "extractive metaphor" (Zimmer 2017, 308), the parallels between mining for stones and minerals and digital mining are not merely allegorical. The current valorization of synthetic or lab-made gemstones or the move toward fully automated mining and polishing operations, for example, blurs the distinction between automated and human-led mining. These changes herald profound transformations for miners across the world and further signal a renewed moment of intensified and expanding extraction (Mezzadra and Neilson 2017). ${ }^{2}$ However, this seemingly limitless and intensified moment of extraction, coupled with the sudden public interest in blockchain technology, raises important concerns for the future organization of digital mining and the effects of capital concentration on the displacement and lack of political sway of mining communities.

Historically, Fernando Coronil $(2000,363)$ argued, the territorial and productive forces of organized capital were progressively obscured by the ideological underpinnings of late capitalism, premised in the concealment of processes of domination and exploitation. Rather than being rooted in spectral forms of value creation, disjointed from its productive core, this article proposes to examine digital mining as a form of labor. Though an obscure and still incipient facet of this digital economy, the work of mining powers and secures transactions across blockchains: public (and sometimes private) distributed digital ledgers. And yet, for all the attention garnered around this technology, which has enraptured digital activists, investors, and speculators alike and made its way into the wider public under the guise of the Bitcoin network, little attention has been paid to the work of miners in this context. If transparency and opacity require specific technologies to be recognized as such (Ferry 2016a), this article critically examines the workings of this labor system by analyzing the categories by which it is represented and how they operate. As a form of labor, I suggest, the value of digital mining lies not in the finished good but in its mode of productive, meaningful action (Graeber 2001). By mining, as in Locke's theory of property quoted earlier, the labor that goes into the object produced becomes part of oneself, and one can claim that thing as one's own. As one crypto-miner put it, explaining why he had ventured into mining operations, "I feel like I'm contributing by mining."3

Drawing from interviews with blockchain advocates, developers, and cryptocurrency enthusiasts; participation in online and offline discussions; and a survey with small-scale crypto-miners, this article brings an ethnographic inquiry into the distinction between abstract and concrete work, material and immaterial labor. By looking at crypto-mining, second, this study suggests that the work of digital miners can inform contemporary debates about changing conceptions of labor in a digital economy. If a yardstick for measuring the success of technology companies is premised in the concealment of the human element behind the operation of algorithms and mathematical codes (Irani 2015), this article illuminates the social and human contribution to powering the blockchain ecosystem and materializing the alternative "dreams" of decentralization and disintermediation (Swartz 2017). Finally, this article suggests that the system of digital mining was founded on unresolved democratic principles, the significance of which anthropology is well equipped to examine.

\section{The materiality of blockchain rocks}

Bitcoin and other digital or cryptocurrencies are under increasing public scrutiny, mostly on account of their volatility and the promises they contain for a decentralized, open, and secure monetary system. Their open source foundation and blockchain technology, compounded by a meteoric valuation, have led to the proclamation of a new era of decentralized networks premised in a form of cryptographic evidence, and taking openness, transparency, and security as central tenets (Narayanan et al. 2016; Tapscott 2016). There are today more than sixteen hundred different digital cryptocurrencies or altcoins in circulation, for a total market capitalization of approximately US $\$ 400$ billion. ${ }^{4}$ Its most popular materialization, bitcoins, are the most widely circulated digital currency in the world, with a current market capitalization of US\$148 billion (as of May 2018), compared to US\$27 billion in April 2017 (cf. Hileman 
and Rauchs 2017). As an electronic, peer-to-peer payment system, Bitcoin was founded on three basic principles: decentralization, transparency, and security. These principles correspond grosso modo to its "peer-to-peer" design, its blockchain structure where all transactions are made public, and the basic cryptographic operation that hides each transaction behind a combination of numbers and text. Beyond the internal logic of distributed accountability, and as a flipside of its unregulated and decentralized principles, there are vulnerabilities external to the system itself, such as in the event of frequent money exchange scams, loss of access keys and accompanying "burning" of assets, or inadequate privacy (Grinberg 2011; Karlstrom 2014; Maurer 2017; Reid and Harrigan 2013). "By wishing away infrastructural work," Lana Swartz $(2017,92)$ suggests, "the bitcoin ecosystem has become as centralized and mediated as existing systems." 5

For those without a programming, computer science, or systems analysis background, the language of the blockchain technology may sound esoteric and off-putting. In ways similar to a plot of land in conventional mining, the core of the bitcoin architecture is the block. Every successful operation is registered and timestamped in these blocks to avoid double transactions, a system or protocol known as proof-of-work, to which I return later. Suffice to say that not unlike finding a nugget of gold or a rough diamond in nature, the proof-of-work system takes place once a value is found or a puzzle is solved. ${ }^{6}$ In addition to the infrastructure of blocks, the basic component of this digital labor is the hash. Hash could be compared to gravel in mining operations - a concentrate of stones that need to be excavated to produce the desired mineral. Though made of data, rather than an aggregate of minerals, hash is a composite sequence of bits. To follow the analogy, hashing is similar to sifting through gravel - the labor of artisanal miners - or grinding diamond-rich ore - as done much more effectively in industrial mines. ${ }^{7}$

The cryptocurrency ecosystem opens up a number of questions of anthropological relevance surrounding money, trust, and materiality. However timid, most approaches to this system stress the swift rise (and crash) in value and its inherently speculative nature. The Bitcoin network and the universe of digital cryptocurrencies have only recently been open to anthropological and sociological inquiry (e.g., Dodd 2017; Ferry 2016b; Karlstrom 2014; Maurer, Nelms, and Swartz 2013; Nelms et al. 2017; Swartz 2017, 2018). Maurer, Nelms, and Swartz (2013, 273) offer a suggestive analysis of Bitcoin's semiotic "practical materialism," a form of "materialism that emphasizes all that is inherent to the code" and, by extension, to the currency itself. In what the authors call "digital metallism," trust, community, and labor itself would be hardwired in metaphors, tropes, and discourses of work and mining. And yet, the code does not inhere simply in people's minds or speech. Even embedded in the code, the system works in real, concrete ways. Though entrusted in algorithmic operations, the human labor power of miners, I contend, is a cornerstone of the Bitcoin protocol, working alongside, at times against - but not outside - automated mining. This is not to dismiss the semiotic claims to the intrinsic value of bitcoins, which hold sway among crypto-enthusiasts much like similar analogies among gold actors examined by Ferry (2016b). However, bitcoins are much more than a medium of exchange or value stored in the virtual world, and a reluctance to taking seriously its human and material underpinnings places the labor of powering the network at odds with the human economy of miners, as I hope to make clear. If Dodd $(2017,45)$ is right in that a view of Bitcoin as simply an embedded trust in code "misses some crucial aspects of the reality of Bitcoin's actual operation, and replicates the ideology behind it," I would be wary of positing the task of protecting the "value of money," usually assigned to central banks or some other institution, as falling squarely "to machines" (41). If nothing else, the 2013 price manipulation by bot-powered trading in the now defunct Mt. Gox exchange in which one actor single-handedly hiked the conversion rate of bitcoins from $\$ 150$ to $\$ 1,000$ USD (Gandal et al. 2018) should admonish us against the risk of entrusting value with automated processes and seemingly inscrutable algorithmic operations.

Here I consider the cryptoecosystem as a form of labor, performed in the work of inscribing, registering, and politically organizing mining operations, inevitably embedded in social relations. A strict focus on the exchange of currency rather than its production, I suggest, fails to account for its thriving economy of miners, the ongoing "arms race" for mining technology, the equipment makers, and an array of exchangers and transaction processors 
that have cropped up in its stead. Increasingly perceived as a standard-bearer of the future of blockchain technology, as an asset and stock that people buy into, a currency and commodity, the Bitcoin ecosystem cannot be meaningfully separated from its different components, the keystone of which is mining. This much was apparent in June 2017, when a group of blockchain enthusiasts gathered in Geneva, Switzerland, for a kick-start meeting of Blockchain 4Good Rocks!

This subgroup is part of the fourteen-hundred-strong members of the Blockchain Meetup assembly in the city. The larger group fosters discussions on market-based applications of the blockchain technology, including the delivery of computing capacity or logistics and supply chain solutions. The smaller gathering brings together blockchain activists that recall the first generation of hackers; libertarian, anticensorship, and privacy-enhancing "cypherpunks"; and cryptographic militants (Coleman 2013; Swartz 2018). The kick-off meeting featured presentations of blockchain applications "for good," including leading proponents of Procivis, a provider of the "self-sovereign" personal data and government-trusted digital identity platform Giveth.io, an open source platform for charitable giving and transparent donations, and Everex, an Ethereum-based financial services company specializing in cross-border payments and a currency remittance pilot program between Thailand and Myanmar. For the thirty-odd people in attendance, coming from different parts of Switzerland where the "blockchain revolution" has been taking shape around the "cryptovalley" of Zug, there was a bewildering sense of curiosity and a vivid appreciation that something new was dawning on the horizon. What that something new would look like, though, was not entirely apparent, and the discussion delved into a passionate disagreement over the blockchain. Was it a decentralized network? A public digital ledger? And how could it be used for good? Yet, for these blockchain believers, the discussion of the blockchain's utility - its real-world application — was dissociated from the labor required to power it. What exactly makes it work, or, more to the point, who are the digital miners?

\section{Digital miners}

In a bitcoin forum, user Inception1337, presented as an eighth-grade student of a "cyber school," posted a tongue-in-cheek essay on December 10, 2013: "Short on money? Want to make some extra dough for the holiday season? Try Bitcoin! Bitcoin is an online digital currency. ... But, did you know that you can create these coins, and help the network at the same time? This is a process called 'mining,' and you can do it too!" After recommending what equipment to buy, how to locate the miner's IP address, and joining a mining pool where resources are shared, the "eighth grader" concluded by explaining how to "make" a miner:

Get an account at btcguild.com, and make a worker. ... Then connect the miner using the stratum address. Also, be prepared for your earnings to slowly go down. There is a new difficulty every week, which it [sic] means it's harder to get a coin. But, don't worry! The price is going up. 2 years ago, it was worth $\$ 6$ ! It's gone up over 100x since then! Also, that's the other thing. The price may crash. If it does, don't fret! The price will be back up over that in no time! ... There may be some risks, but they're like any job. I've actually paid off the miner in less than 1 month! So, Bitcoin can really help our economy. It can help the WORLD economy!

The original post on the bitcoin "subreddit" (http://reddit/r/bitcoin) received more than nine hundred comments. In all likelihood, this was not a text written by an eighth-grade student, as, it turned out a few months later, the user went on to found yet another cryptocurrency, ImperialCoin. But the point - which seemed to elicit positive reactions - was that mining in the age of dedicated machines was so simple that even a kid could do it. Not unlike the hacking communities studied by Coleman (2013), humor and wit punctuate interactions among miners. These are "self-determined and rational individuals who use their well-developed faculties of discrimination and perception to understand the 'formal' world - technical or not - around them with such perspicuity that they can 
intervene virtuously within this logical system either for the sake of play, pedagogy, or technological innovation" (Coleman 2013, 7). But what does it mean to "make a worker" and have it "pay off"?

As we have seen, the most overlooked element of the digital technoindustry - mining - is the one that validates the system and makes it possible ("helps the network," as in the preceding citation) and could in the same way possibly undermine it. To be sure, miners discover new coins by solving exceedingly complex mathematical problems and contribute to the strength of the system by verifying all transactions. Both actions, discovery and verification, constitute mining. Based on decentralized nodes of hardware processing power, individual users ("miners") deploy their computer hardware to mine cryptocurrencies, thus increasing the processing power of the network as a whole.

Most cryptocurrency enthusiasts with whom I have interacted have at times expressed some ambivalence toward mining. Some crypto-miners conveyed skepticism that exponential costs would render mining too expensive and the equipment obsolete ("Too late to get into the mining game, bro" was one response to a prospective bitcoin miner in 2014), ${ }^{8}$ whereas others quipped that there would not be a race for power if it did not make sense economically. In any event, running a bitcoin mining operation can be prohibitively expensive, and miners' profit margins are reduced further with the increased difficulty relative to available computational power, hardware that quickly becomes outdated, the cost of electricity, or new taxation over capital gains.

Crypto-miners are still a relatively unknown community. In a 2014 study, 96\% of participants surveyed were male and between the ages of 18 and 34 years (69\%), with the majority based in the United States (50.7\%) and Europe. ${ }^{9}$ It is also a fast-changing population, particularly with the rise in popularity (and value) of cryptocurrencies. In two surveys I conducted in January 2018 among 115 crypto-miners (altcoin and bitcoin miners) from fifteen different countries, the overwhelming majority of current miners had begun mining over the previous year $(75 \%$ for bitcoin miners and $85.5 \%$ for other altcoins). ${ }^{10}$ In addition to bitcoins, the thirty-one respondents who were bitcoin miners also tended to mine Litecoin or Ethereum (35\%), with an additional 25\% spread evenly between Dash, Bitcoin Cash, and ZClassic. Some respondents sell their hashing power on one of the largest crypto-mining marketplaces, NiceHash, a cloud mining company.

Altcoin miners, on the other hand, are less firm on the specific currency of choice to mine. Although bitcoins, litecoins, and ethereum constitute more than half of mined coins (54\%), with a marked preference for the latter (34\%), altcoin miners' choice of coins seemingly oscillates in accordance with price fluctuations. One participant, for example, claimed to have mined at least fifty-five different cryptocurrencies since November 2017. Others mined their "own currencies" or a mix of coins through cloud computing (10\%). Mining hardware was diverse, ranging from a personal computer or "gaming PC" equipped with a basic GPU mining rig through larger operations (e.g., “ 1000 i5 machines"). Accordingly, mining can have a hobby-like, experiential quality ("I'm currently using my single Asus r9 390x gpu, smallest operation to get my feet wet and see if I want to expand my rig [I do]") to more technically challenging malware solutions ("Botnet of computers owned by tech support scammers"). In contrast to bitcoin miners, last-generation GPU mining rigs were still used by $62.5 \%$ of respondents, with only $12.5 \%$ using the more advanced ASIC equipment.

For the most part, altcoin mining is "a hobby ... this at least makes some [money]," and not fully professionalized ("It's fun to dick around with," one respondent suggested, whereas another said, "I wouldn't trust it to be my full time gig," despite being a "pretty great way to make side income"). One user recollected his inability to determine "where the hobby ends and the OCD begins." Scalability and the inevitable move to more expensive, dedicated equipment for additional hash power will determine the fate of some miners ("Siacoin moves to ASIC miners in 6 months, so it's short term for me to find what I can and hold to see if it goes anywhere"). One respondent expressed his pessimism: "I live in [an] apt. If I try to run 10000000 GPUs it will burn down. I can maybe [have] 10 in one room on one circuit breaker safely for example. How do I ramp up? The heat is another problem. My current set up heats my entire second floor. What do I do in summer time?" Most concerns expressed included 
price or market manipulation (21\%); energy consumption (17.52\%, with one respondent claiming that mining was "a great way to keep my bedroom warm in the Winter"); and, in equal proportions (13\%), the risk of scams, mining centralization, and regulation. Other miners voiced concerns over profitability rates and low returns; the risk of fire; overpriced equipment; the use of malware-infected private computers, or botnets; and the advent of quantum computers. Overall, though, the future holds promise:

I primarily use it as a way to collect new alt coins that I don't have yet. I have no confidence in any particular coins but I do believe crypto is going to have a very good general future, so I want to own small amounts of every coin I can get my hands on and mining has been a great way to do it. I'm not concerned about current income but rather about holding onto lots of little future lottery tickets.

Though "the benefits of blockchain technology add potential that we can only wait and see unfold," one user claimed that crypto-mining "will survive only if it proves to have real utility." Other participants, however, were more enthralled with the experience ("I love it"). One participant claimed that "it's been an amazing experience that I wish I had been part of sooner," whereas another "would love to do it full time" if it turns out to be "viable." More broadly, these crypto-miners present a dynamic understanding of work, defined in a spatiotemporal shift from, first, mining as hobby to, second, considerations of its viability and low returns and, third, the belief in its future utility and a utopian return to postwork play.

In common, bitcoin and other crypto-miners allocate their mining labor in pools, where computational power can be shared for optimal rewards and greater likelihood of finding a new block. According to survey respondents, only $8.4 \%$ and $6.4 \%$ of crypto-miners and bitcoin miners, respectively, did not belong to any mining pool. As a supposedly egalitarian space for distributing computational resources, mining pools are a key component in mining labor. In many regards, mining pools recall workers' trade corporations defined by the equality among members, shared control over production, and the relative autonomy of the collective (cf. Sewell 1980). This speaks well to the "democratic" processes at the heart of the labor of digital mining, the principles of which have come under attack by an increasingly centralized, corporate mining industry, to which I now turn.

\section{Blockchain in the mines: The geography of crypto-mining}

Heralded by advances in big data and the expansion of transparency mechanisms, the mining industry tentatively threads the risks of added scrutiny with the possibilities offered by blockchain technology. The "potential to be able to account for everything, since anything can be entered into it" (Maurer 2017, 222), has captured the imagination of blockchain enthusiasts and investors alike. In the latest fad of "incorporative blockchain applications" (Swartz 2017, 87), Fortune magazine suggested that the "diamond industry is obsessed with the blockchain" (Roberts 2017). In a widely reported initiative led by Everledger, a London-based company, blockchain technology would be used to create a global registry for diamonds. By digitally certifying diamond origins - between 1.6 million and 2 million have been cataloged thus far - the promissory note of this technology would seemingly put an end to conflict diamonds, notwithstanding the more than 100 million carats of rough diamonds mined every year and recent controversies regarding the provenance of "ethical" diamond providers. Ajay Anand, founder of Rare Carat - often glossed as the "Kayak" for diamond buyers — is elated with the capabilities that a working partnership with Everledger would entail. While the company he created already deploys IBM's Artificial Intelligence Watson technology to compare prices across retailers, he describes the "blockchained" diamond as "already hav[ing] a history associated - name, states, times, even shipping air bills, from the mine to the certification [agency] and on to the consumer" (interview, November 15, 2016). With a history "embedded in the blockchain," he admits, the price of diamonds "certified by Everledger ... will go up." Everledger's blockchain is still shrouded in mystery, and at least one blockchain developer I talked to, currently working on a blockchain project of gemstone provenance 
for a gemological laboratory, suggested that there are considerable technical limitations and a frustrating opacity to the enforcement of consensus and control mechanisms in Everledger's blockchain (personal communication, May 2018).

Similar issues cast doubt on pilot projects designed to map out mineral supply chains with private instances of the blockchain technology, including De Beers's Tracr initiative. At the Organisation for Economic Co-operation and Development's (OECD) Forum on Mineral Supply Chains held in Paris in April 2018, I could verify that blockchain was on everyone's lips, and with reason. The traceability of minerals would provide "the most obvious use case for blockchain," despite "some concern about how such an approach could be commercially viable" (Hardy 2018). At the crux of the logistical hurdles of digitally recording every mineral and metal lies an adequate or nonexistent system of incentives capable of fostering mass collaboration. Although few details are publicly available, industry-led blockchain initiatives are premised on pre-mined tokens and private computing power rather than distributed mining or systems of rewards and incentives that would implicate miners - artisanal or digital. The notion of centralized or permissioned database locations, which is to say, who controls access and dissemination of data, is also at odds with the principles of distributed accountability, the "democratic" processes of securing consensus, and the decentralized computational power within the blockchain network. In other words, private-led blockchain-based initiatives based on the stake in the network may push small-scale (crypto) miners and, by extension, artisanal miners out of the system of rewards and incentives in place. This exclusion and the risk of intensifying the digital divide between Global North and South represent the biggest challenges for multistakeholder blockchain initiatives across the mineral supply chain and are compounded by the growing centralization of blockchain mining, as we will see next.

Over recent years, the economy of digital cryptocurrencies has been moving away from individual, accessible, and relatively affordable mining toward industrial-like, corporate, and largely unaccountable mining conglomerates. This process of mining centralization is manifest in at least three different ways, first among these in the manufacturers of mining equipment. The appearance of new machines specifically designed for mining (commonly known as application-specific integrated circuit, or ASIC) reduces the profitability of former hardware while granting an exceeding power to its manufacturers. Different cases have been registered where unscrupulous manufacturers failed to deliver the equipment or simply ran away with the money, namely Butterfly Labs, a company shut down at the request of the US Federal Trade Commission in September 2014. Despite criticism of power-hungry manufacturers or the rising price of gaming equipment as a result of a run for GPU mining rigs, most miners are not particularly concerned with the centralization of hardware manufacturing (Hileman and Rauchs 2017). Second, the location of computational power and, third, who controls it offer far more profound concerns in terms of the perils of centralization in the cryptocurrency ecosystem.

For the most part, the location and ownership of crypto-mining purpose-built, large-scale mining facilities remain elusive, with only anecdotal evidence and punctual media accounts allowing for a composite picture of these bourgeoning high-density centers for concentrated computing power. According to the lead developer of a blockchain-based decentralized cloud storage solution, access to "secret ASICs" based on "rumor and previous relationships" remains the crucial question in the development of cryptocurrencies, with "an informal underground industry ... set up around secret mining" (Vorick 2018). Given the inordinate amounts of energy required to power billions of calculations per second, crypto-mines - and bitcoin mining facilities in particular - have fallen under public scrutiny, with some estimates calculating the total amount of energy used to be roughly 42 tWh per year, for a total annual carbon footprint of 20,768 (kt of $\left.\mathrm{CO}_{2}\right) \cdot{ }^{11}$ It is not surprising, therefore, that access to low-cost electricity and cold regions, which can offer natural cooling solutions, are important factors in the choice of crypto-mining locations. Heretofore, most large-scale mining facilities have been located in China. According to Hileman and Rauchs $(2017,94)$, a "significant concentration can be observed in the Sichuan province, where miners have struck deals with local hydroelectric power stations to access cheap electricity." Coal-powered, energy-hungry 
mines echo dystopian images of a bygone industrial era. An account of one of the oldest bitcoin mines, owned by a Beijing-based company, Bitmain, resonates well with this gloomy and enigmatic world: "Fifty Bitmain staff ... watch over eight buildings crammed with 25,000 machines that are cranking through calculations 24 hours a day. One of the buildings is devoted to mining litecoin, an ascendant cryptocurrency. The staff live on-site in a building with a dormitory, offices, a canteen, and a repair center. For recreation, they play basketball on an unfinished cement court" (Wong and Simon 2017).

In contrast to the original hobbyist days of running mining operations in domestic makeshift arrangements, new mining configurations capable of offering more effective cooling systems have been proposed. Kampl (2014, 7) describes an ASIC $500 \mathrm{~kW}$ mine built in Hong Kong in 2013. This fully automated cluster of high-performing mining equipment would run "silent and dust free," requiring "only a single person to check on the system every few days" (Kampl 2017, 7). Unlike the loud humdrum of other mining facilities, this "proof-of-concept for a new era of high density computing" would be kept clean by a "Roomba iRobot" (Kampl 2014, 7). Moreover, as mines pop up across the world, from Russia to Canada, Iceland (Popper 2013), and Sweden (Economist 2015), it seems clear that the geography of crypto-mining is changing accordingly.

In only a few years, crypto-miners have been increasingly organized into dedicated mining pools (combining their total power to solve a block), mining rigs (running multiple computers), and cloud mining (by renting equipment from private companies). In this move toward collective and pooled mining, there can be an implicit loss of control and oversight over the labor process: the miner may well be mining but have no idea as to what corresponds to one's share. It is hard to quantify the pervasiveness of these schemes, but it is indicative that some cloud mining companies that have flourished in the Bitcoin ecosystem over the last few years have used similar ploys. Following a guilty plea, crypto-mining firm GAW Miners was accused and charged by the US Securities and Exchange Commission of defrauding its customers by offering the services of "virtual miners" - with hash power it did not own. This speaks, also, to the modality of cooperation implicit in pooled mining, whereby individual miners transfer the control over full nodes - and thus the burden of proving work - to the centralized pool (Swartz 2018, 636), further consolidating the move to industrial mining. It is to the problem of proving work, and the importance of labor more broadly in an increasingly capital-intensive mining regime, that I look next.

\section{Taking over the means of automation: Labor and consensus}

There are two main protocols for ensuring consensus across the cryptoecosystem, known as proof-of-work (PoW) and proof-of-stake (PoS), aside from multiple other variations (Zheng et al. 2017). Each entails different challenges for the future of blockchain technology and its governance. Defining which solution to power the blockchain has become the fodder for much debate in cryptospecialized online forums, focused primarily on the security of the network, the energy consumed to maintain it, and the scalability of blockchain-derived applications. Although a majority of cryptocurrencies have currently adopted nonmineable PoS protocols, the two largest cryptocurrencies by market capitalization and full-fledged blockchain networks - Bitcoin and Ethereum - are based on PoW protocols, rewarding miners who solve complex algorithms and validate transactions with new coins. In common, both protocols rely on the maintenance of consensus, which is to say, on how distributed systems should operate across trustless and unmediated transactions.

For its detractors, the PoW protocol is eminently wasteful, as it entails vast swathes of time, energy, and electricity to control mining power. PoS, on the other hand, is presented as energy-efficient, as it does not require the full strength of computational power to generate new coins. Rather, the solution to power PoS cryptocurrencies depends largely on early investors and currency holders maintaining control over the network since the coin's public inception. The reduced risk of centralization would be outweighed by an emergent plutocracy, where the power over the network - and the likelihood of manipulation or attack — is tied to the size of one's wallet. Unsurprisingly, corporations seeking to develop private blockchain-based applications have mainly adopted PoS. 
In a PoW system, on the other hand, those holding labor power - digital miners - build the blockchain in the process of verifying the system and avoiding the threat of double transactions. With ever-increased centralized mining operations, including ASIC chip manufacturing, PoW solutions run the risk of being turned into an oligarchy of capital-intensive computational power and energy expenditure. However, the importance of hash-based PoW, as a measure of the processing power of the entire network - which is to say, the speed of mining or the number of calculations mining hardware can make per second - is manifold. First, the block chain establishes a temporal order, an official record of each operation marked in time. Second, there is a reward or incentive for every block that is found, which is how most coins are generated. ${ }^{12}$ Finally, as I suggest here, the strength of a system based on the work of computing force - notwithstanding the significant risks highlighted - holds the potential for democratic control.

The PoW concept predates cryptocurrencies and was proposed in the early 1990s to combat spam mail (Dwork 1993) and ensure the security of communication (Jakobsson and Juels 1999). At the root of the system's algorithm-based operations and the adaptive difficulty of its puzzles is the idea that "proof-of-work is essentially one-CPU-one-vote" (Nakamoto 2008, 3), which is to say that mining rewards are within reach, provided you have the luck, energy, and computational power to solve these puzzles. Importantly, PoW also makes plain that the predictability and stability of the system are only possible by a consensual, majority control represented by the longest blockchain. Democratic participation, then, is defined in the collective product of labor that requires the greatest effort to produce. Conversely, a rogue majority control over the network's hash rate could possibly entail its collapse by reversing transactions or failing to prevent double spending.

It is here that control over the network power becomes paramount. As we have seen, PoW and valid transactions are registered in the blockchain, which provides the system's temporal coordinates. However, the cost of running a decentralized, cryptographic currency secured by PoW can be suddenly undermined if one of the existing mining pools takes a dominant share of the total computational power. A rogue $51 \%$ takeover attack of this kind would corrupt the blockchain and the mutually agreed upon and consensual work that has been put into it. This uncertainty and potential vulnerability - the possibility to "fake and publish an 'alternative history"” (Barber et al. 2012, 405) - stems in great measure from the dynamic nature of this process (shifting computational power, for example) and the tacit admission that miners would have to voluntarily decide to go rogue against the system in place. In a notable episode in 2014, one of the most popular bitcoin pools (Ghash.io) witnessed an exodus of its miners after the pool's hash share threatened to cross the $51 \%$ mark. Moreover, a group of analysts questioned (Becker et al. 2013, 149), "What if a large number of CPU-owners vote against the system?" This scenario, which they call "Occupy Bitcoin," is at once an ever-present threat to the system but proof of its vitality as well. Paradoxically, the dangers to the long-term structural stability and security of the Bitcoin system augment exponentially if participation decreases with the dearth of miners and verifiers; in other words, if circulation drops and deflationary pressure mounts, the system becomes too weak and vulnerable to defend itself from diverted computation power. ${ }^{13}$ At a more fundamental level, as Kroll, Davey, and Felten $(2013,7)$ remind us, "consensus about the rules is a social process," and there needs to be a shared "understanding of what is allowed, so that the rules can be encoded into the software that each participant uses." Despite the risks of mining centralization, politics is not removed from its technological underpinnings, or "that the reality of Bitcoin - its social reality — is at odds with the theory behind it" (Dodd 2017, 46). In fact, it is the nature of the conflict itself that grants the work of mining its political character.

Proving one's work, more broadly, should serve as a stepping-stone toward a shared digital commons, one that sidelines capital-intensive mining operations or the rapid professionalization of mining and opens up for scrutiny the rules governing the technology. It may be necessary, also, to return to the "essential but boring work done by custodians of information systems" (Swartz 2018, 634) by creatively coming up with different incentives to conjure the technoimaginary of a decentralized and unmediated system where ethical labor practices take root. One way of achieving just that would be to render PoW algorithms socially valuable, not by ultimately wasteful and 
mathematically arbitrary hashing, but in channeling computing power toward useful operations - whether they are decentralized, modular mass collaborations in the real world or scientific endeavors.

\section{Conclusion}

At the time of writing, the cryptocurrency market was on the verge of yet another crash, with prices taking a nosedive in less than twenty-four hours. Perhaps in jest, suicide hotline numbers were being posted in at least one cryptocurrency discussion forum. Rather than accounting for the volatility in price fluctuations driven by a strict concern over electronic monies, the work of miners allows for overcoming the technological abstraction in concrete human action, creating value in "the way that actors see their own activity as meaningful as part of it" (Graeber 2001, 76). Imagining a techno-future of unmediated relationships in an open, decentralized system of distributed accountability should not obviate the potential for valorizing the work of these digital economies, whether led by human or nonhuman automated processes.

Miners are at the crux of this digital economy in two related ways: just as much as they commit the sheer strength of their computing power to a blockchain, they also keep the network secure, preventing the potential threat of turning this power on its head by reversing the legitimate and consensual record of transactions. To be sure, transaction fees are poised to replace existing rewards in the future. But corporate-led attempts to create private blockchains, as in the recent push for nonmineable Ripple cryptocoins, where history can be reversed by removing PoW and consensus is placed squarely in the hands of major banks and private companies, should be seen as attempts to depoliticize and dehumanize the labor of this economy. Granted, unaccounted control over hash power, or the one thousand or so "bitcoin whales" - large bitcoin holders that control and could potentially manipulate over $40 \%$ of the bitcoin market - represent important risks to this emerging technology. As I have suggested here, however, cryptocurrencies have a foundation myth, a clear sense of historicity, and a regime of technoscientific valuation that posits time and labor at the heart of their production.

This is not to suggest that all miners share a commitment to working toward a community-based, ideologically driven vision of decentralized collaboration or that digital labor is the same across the world. In fact, cases abound of opportunistic, profit-seeking behavior, and the work of mining remains itself subject to the sociopolitical forces and legal restraints central to the making of emergent forms of unfree labor (Calvão 2016). And yet, with epochal transformations in technological, socioeconomic, and legal conditions, crypto-miners have shown to be remarkably resilient to ascribing meaning to the work they do, whether they are under the strenuous conditions of a mining warehouse in China or in a libertarian-inspired mining rig operation of a San Francisco apartment. In the end, the crypto-system may well be the digitized version of the world we already live in, with the many governed by the few, profits over purpose, and the hope that a new labor paradigm may be necessary and in the making. Attention to the politics and technoscientific valuation of crypto-mining labor is one stop on the way to understanding the new communities emerging in its stead.

\section{Acknowledgments}

The author thanks the many interlocutors who volunteered their time to make this research possible. I also wish to acknowledge the helpful suggestions and discussions with Emily Benson, Matan Ilan Shapiro, Simon Fuerstenberg, Claudia Cruz Leo, Peter Graif, and Gretchen Bakke as well as the constructive criticisms of two anonymous reviewers. Last, I thank Brandon D. Lundy and the editorial team of Economic Anthropology for their support.

\section{Notes}

1 Throughout the text, I capitalize Bitcoin when referring to the concept, protocol, or network as a whole, whereas bitcoins is used to describe the monetary unit of account. I avoid abbreviations (BTC or XBT) for ease of reading. I use Ethereum to denote both the blockchain platform and its value token, Ether. 
2 The materiality of natural resources has been the object of a renewed engagement in anthropology, from bodies to infrastructures, labor and material qualities (e.g., Calvão 2013; Richardson and Weszkalnys 2014; Rolston 2013). Pushing extractive boundaries — from the depths of oceans to outer space, from raw data to the body itself — mining has also enabled new forms of currency to emerge, including genetic material and troves of information in bits and bytes. However, this seemingly limitless and intensified moment of extraction introduces new questions on dispossession, displacement, and the concentration of capital (Calvão 2011; Smith 2011).

3 Unless otherwise noted, citations are taken from an online survey of crypto-miners conducted in January 2018.

4 Coinmarketcap.com registers 1,610 cryptocurrencies (as of May 2018). Given that some cryptocurrencies (mineable or not) are pre-mined and made available in so-called initial coin offerings, the total circulating supply artificially increases the market value of these currencies.

5 As I could attest from my own experience buying bitcoins in June 2011. I had to physically mail a money order to an online bitcoin supporter, Morpheus, to then have my Mt. Gox account credited on June 18. The following day, Mt. Gox —-the largest and most respectable bitcoin exchange until its demise in 2013 - registered the first big flash crash, with bitcoins being traded at less than 0.01 cents to the dollar. This crash — known today as the first big bitcoin heist—was followed by a massive leak of Mt. Gox's account database.

6 In more technical terms, this means a function producing the required number of zero bits in relation to PoW in the block. Once a valid result has been found, the work is registered and cannot be altered without entirely changing the work that preceded it and the blocks that followed. As blockchains expand and are subsequently linked to each other in a massive blockchain, the stability of the system relies on this principle.

7 The basic cryptographic hash function is to generate numbers from strings of text, which, in a mix of computational power and luck, would yield the valid result. As Becker et al. $(2013,136)$ put it, Bitcoin "users constantly participate in a lottery, and each user's chance of winning is proportional to the computing power he is willing to invest" in proportion to the total network power.

8 User 1776 m8, http://reddit.com/r/BitcoinBeginners, November 19, 2014.

9 This study was led by a graduate student in the Informatics department at UC Irvine (see https://bitcointalk.org/index.php?topic=486149. $\mathrm{msg5354626 \#}$ msg5354626).

10 Participants were mainly based in the United States, followed by Canada, Australia, England, and Sweden. The survey was conducted online in specialized cryptocurrency and altcoin fora, including bitco.in/forum, forum.ethereum.org, Cryptocurrencytalk.com, forum.bitcoin.com, and different subreddits, including r/altcoin, r/cryptocurrency, r/cryptominingreddit.com, and r/bitcoinmining.

11 Data from https://digiconomist.net/bitcoin-energy-consumption. For comparison, the amount of electricity consumed in all African nations but Algeria, Egypt, and South Africa is less than the electricity consumption required to power Bitcoin mining operations globally (cf. https://powercompare.co.uk/ bitcoin/).

12 The current reward is 12.5 bitcoins, although it is poised to be halved progressively as the difficulty of the puzzles required to solve them is increased. The level of difficulty oscillates in accordance with the available hashing power of the network in order for each block to be mined every ten minutes, or the time it takes for every transaction to be broadcast, collected, registered, and broadcast again for the network's approval.

13 One of the suggestions to avoid this situation grants miners greater autonomy for "private checkpointing," or a mode of legitimized verification based on miners' "own remembered history" (Barber et al. 2012, 406). This would result in a system of "implicit voting" where seasoned miners would be given an added vote of confidence to prevent attacks and loss of value and decide which way the network should proceed in case of a partitioned blockchain.

\section{References}

Barber, Simon, Xavier Boyen, Elaine Shi, and Ersin Uzun. 2012. "Bitter to Better-How to Make Bitcoin a Better Currency." In Financial Cryptography and Data Security, edited by Angelos D. Keromytis, 399-414. Heidelberg, Germany: Springer.

Becker, Jörg, Dominic Breuker, Tobias Heide, Justus Holler, Hans Peter Rauer, and Rainer Böhme. 2013. "Can We Afford Integrity by Proof-of-Work? Scenarios Inspired by the Bitcoin Currency." In The Economics of Information Security and Privacy, edited by Rainer Böhme, 135-56. Berlin: Springer.

Calvão, Filipe. 2011. "When Boom Goes Bust: Ruins, Crisis and Security in Megaengineering Diamond Mines in Angola." In Engineering Earth: The Impacts of Megaengineering Projects, edited by Stan Brunn, 367-82. Dordrecht, the Netherlands: Springer.

_. 2013. "The Transporter, the Agitator, and the Kamanguista: Qualia and the in/visible Materiality of Diamonds." Anthropological Theory 13(1/2): $119-36$.

- 2016. "Unfree Labor." Annual Review of Anthropology 45: 451-67.

Coleman, Gabriella. 2013. Coding Freedom: The Ethics and Aesthetics of Hacking. Princeton, NJ: Princeton University Press.

Coronil, Fernando. 2000. "Toward a Critique of Globalcentrism: Speculations on Capitalism's Nature." Public Culture 12(2): 351-74.

Dodd, Nigel. 2017. "The Social Life of Bitcoin." Theory, Culture, and Society 35(3): 35-56.

Dwork, Cynthia, and Moni Naor. 1993. "Pricing via Processing or Combatting Junk Mail." In Advances in Cryptology —CRYPTO' 92. 12th Annual International

Cryptology Conference Santa Barbara, California, USA, August 16-20, 1992: Proceedings, edited by Ernest F. Brickell, 139-47. Berlin: Springer.

Economist. 2015. "The Magic of Mining." January 8.

Ferry, Elizabeth. 2016a. "Gold Prices as Material-Social Actors: the Case of the London Fix." Extractive Industries and Society 3(1): 82-5.

—. 2016b. "On Not Being a Sign: Gold's Semiotic Claims." Signs and Society 4(1): 57-79.

Gandal, Neil, J. T. Hamrick, Tyler Moore, and Tali Oberman. 2018. "Price Manipulation in the Bitcoin Ecosystem." Journal of Monetary Economics 95: 86-96. Graeber, David. 2001. Toward an Anthropological Theory of Value: The False Coin of Our Own Dreams. New York: Palgrave. 
Grinberg, Reuben. 2011. "Bitcoin: An Innovative Alternative Digital Currency." Hastings Science and Technology Law Journal 4(1): 159-208.

Hardy, Sam. 2018. "RCS Primer: A Look Back at the 12th OECD Forum on Responsible Mineral Supply Chains." RSC Global News, April 26. http://www .rcsglobal.com/rcs-primer-a-look-back-at-the-12th-oecd-forum-on-responsible-mineral-supply-chains/.

Hileman, Garrick, and Michel Rauchs. 2017. "Global Cryptocurrency Benchmarking Study." April 6. https://doi.org/10.2139/ssrn.2965436.

Irani, Lilly. 2015. "Difference and Dependence among Digital Workers: The Case of Amazon Mechanical Turk." South Atlantic Quarterly 114(1): 225-34.

Jakobsson, Markus, and Ari Juels. 1999. "Proofs of Work and Bread Pudding Protocols (Extended Abstract)." In Secure Information Networks, vol. 23, edited

by Bart Preneel, 258-72. Boston, MA: Springer.

Kampl, Alex. 2014. "Analysis of Large-Scale Bitcoin Mining Operations (or How Bitcoin Miners Make $\$ 845$ Million a Year)." White Paper. http://ww1.prweb .com/prfiles/2014/03/29/11636018/Analysis_of_Large-Scale_Bitcoin_Mining_Operations.pdf.

Karlstrom, Henrik. 2014. "Do Libertarians Dream of Electric Coins? The Material Embeddedness of Bitcoin." Distinktion: Scandinavian Journal of Social Theory 15(1): $23-36$

Kroll, Joshua A., lan C. Davey, and Edward W. Felten. 2013. "The Economics of Bitcoin Mining, or Bitcoin in the Presence of Adversaries." Paper presented at the 12th Workshop on the Economics of Information Security, Washington, DC.

Locke, John, and Peter Laslett. 1988. Two Treatises of Government. Cambridge: Cambridge University Press.

Maurer, Bill. 2017. "Blockchains Are a Diamond's Best Friend: Zelizer for the Bitcoin Moment." In Money Talks: Explaining How Money Really Works, edited by Nina Bandelj, Frederick F. Wherry, and Viviana A. Zelizer, 215-29. Princeton, NJ: Princeton University Press.

Maurer, Bill, Taylor C. Nelms, and Lana Swartz. 2013. "'When Perhaps the Real Problem Is Money Itself!': The Practical Materiality of Bitcoin." Social Semiotics 23(2): $261-77$.

Mezzadra, Sandro, and Brett Neilson. 2017. "On the Multiple Frontiers of Extraction: Excavating Contemporary Capitalism." Cultural Studies 31(2-3): $185-204$.

Nakamoto, Satoshi. 2008. "Bitcoin: A Peer-to-Peer Electronic Cash System." https://bitcoin.org/bitcoin.pdf.

Narayanan, Arvind, Joseph Bonneau, Edward Felten, Andrew Miller, and Steven Goldfeder. 2016. Bitcoin and Cryptocurrency Technologies: A Comprehensive Introduction. Princeton, NJ: Princeton University Press.

Nelms, Taylor C., Bill Maurer, Lana Swartz, and Scott Mainwaring. 2017. "Social Payments: Innovation, Trust, Bitcoin, and the Sharing Economy." Theory, Culture, and Society 35(3): 13-33.

Popper, Nathaniel. 2013. "Into the Bitcoin Mines." New York Times, December 21.

Reid, Fergal, and Martin Harrigan. 2013. "An Analysis of Anonymity in the Bitcoin System." In Security and Privacy in Social Networks, edited by Yaniv Altshuler, Yuval Elovici, Armin B. Cremers, Nadav Aharony, and Alex Pentland, 197-223. New York: Springer.

Richardson, Tanya, and Gisa Weszkalnys. 2014 "Introduction: Resource Materialities." Anthropological Quarterly 87(1): 5-30.

Roberts, Jeff John. 2017. "Crystal Clear Provenance." Fortune, September 15.

Rolston, Jessica Smith. 2013. "The Politics of Pits and the Materiality of Mine Labor: Making Natural Resources in the American West." American Anthropologist 115(4): 582-94.

Sewell, William. 1980. Work and Revolution in France: The Language of Labor from the Old Regime to 1848. Cambridge: Cambridge University Press.

Smith, James. 2011. "Tantalus in the Digital Age: Coltan Ore, Temporal Dispossession, and 'Movement' in the Eastern Democratic Republic of Congo." American Ethnologist 38(1): 17-33.

_ 2017. "Blockchain Dreams: Imagining Techno-Economic Alternatives after Bitcoin." In Another Economy Is Possible: Culture and Economy in a Time of Crisis, edited by Manuel Castells, 82-105. Cambridge: Polity Press.

Swartz, Lana. 2018. "What Was Bitcoin, What Will It Be? The Techno-Economic Imaginaries of a New Money Technology." Cultural Studies 32(4): 623-50. Tapscott, Don, and Alex Tapscott. 2016. Blockchain Revolution: How the Technology behind Bitcoin Is Changing Money, Business, and the World. New York: Penguin Random House.

Vorick, David. 2018. "The State of Cryptocurrency Mining." Sia Tech (blog), May 13. https://blog.sia.tech/the-state-of-cryptocurrency-mining-538004a37f9b. Wong, Joon lan, and Johnny Simon. 2017. "Photos: Inside One of the World's Largest Bitcoin Mines." Quartz, August 17. https://qz.com/1055126/photoschina-has-one-of-worlds-largest-bitcoin-mines/.

Zheng, Zibin, Shaoan Xie, Hongning Dai, Xiangping Chen, and Huaimin Wang. 2017. "An Overview of Blockchain Technology: Architecture, Consensus, and Future Trends." In 2017 IEEE 6th International Congress on Big Data (BigData Congress Proceedings), edited by George Karypis and Jia Zhang, 557-64. Los Alamitos, CA: IEEE Computer Society.

Zimmer, Zac. 2017. "Bitcoin and Potosí Silver: Historical Perspectives on Crytocurrency." Technology and Culture 58(2): 307-34. 